\title{
Preparation of Chitosan Nanoparticles and its Synergistic Effects against Gram Positive and Gram Negative Microorganisms
}

\author{
Krishnaswamy Ezhilan Vivekanandan* (D), Swethavinayagam (iD, \\ Deepika Venkatesan (iD and L. Jeyanthi Rebecca
}

Department of Industrial Biotechnology, Bharath Institute of Higher Education and Research, Selaiyur, Tambram, Chennai - 600 126, Tamilnadu, India.

\begin{abstract}
Chitosan nanoparticles have attained more attention due to its potential applications in food, agriculture and pharmaceutics. Owing to its high biodegradability, and nontoxicity and antimicrobial properties, chitosan is widely-used as an antimicrobial agent either alone or blended with other natural polymers. The purpose of the experiment was to assess the antimicrobial bustle of chitosan based nanoparticles against few medical pathogens by using chitosan conjugated nanoparticles which are prepared based on the ionic gelation of chitosan extracted from the crab shell waste along with tripolyphosphate (TPP) anions. The chitosan nanoparticles characterization was confirmed by the diffraction study and spectral analysis. The size of the nanoparticle was identified using Transmission electron microscopy (TEM). The spectral studies showed a characteristics bands of $-\mathrm{NH} 2$ (amide) at $3450 \mathrm{~cm}^{-1}$ and carbonyl at $1629 \mathrm{~cm}^{-1}$. $X$-ray diffraction (XRD) patterns also indicated two characteristics crystalline peaks approximately at $20^{\circ}$ and $35^{\circ}(2 \theta)$. The antibacterial activity of chitosan nanoparticles against medical important pathogens such as Escherichia coli, Staphylococcus aureus was estimated using minimum inhibitory concentration (MIC). The presence of primary amine groups in repeating units of chitosan grants reveals it several properties like antibacterial activity, antitumor activity.

Keywords: Chitosan; Chitin; Antimicrobial activity; biopolymer; Chitosan nanoparticles.
\end{abstract}

*Correspondence: vivekanandanbiotech@gmail.com

(Received: 09 April 2019; accepted: 30 November 2019)

Citation: Krishnaswamy Ezhilan Vivekanandan Swethavinayagam, Deepika Venkatesan and L. Jeyanthi Rebecca, Preparation of Chitosan Nanoparticles and its Synergistic Effects against Gram Positive and Gram Negative Microorganisms, J Pure Appl Microbiol., 2019; 13(4):2317-2324. https://doi.org/10.22207/JPAM.13.4.47

(C) The Author(s) 2019. Open Access. This article is distributed under the terms of the Creative Commons Attribution 4.0 International License which permits unrestricted use, sharing, distribution, and reproduction in any medium, provided you give appropriate credit to the original author(s) and the source, provide a link to the Creative Commons license, and indicate if changes were made. 


\section{INTRODUCTION}

Chitosan is a linear polysaccharide composed of randomly distributed $\beta-(1-4)$ linked D-Glucosamine (deacetylated unit) and $\mathrm{N}$-acetyl-D-glucosoamine (acetylated unit). It is made by treating the chitin shells of the shrimp and other crustaceans with an alkaline substance, like sodium hydroxide. Chitosan and its derivatives have shown broad range of applications due to their antimicrobial and antifungal activity. It is made by treating the chitin shells of shrimp and different scavengers with a basic substance, similar to sodium hydroxide. Chitosan has conceivable biomedical employments. It may be utilized in horticulture as a seed treatment and biopesticide, helping plants to fend off contagious diseases. The chitosan is a characteristic nontoxic biopolymer by the removal of acetate in chitin ${ }^{1}$.

The chitosan based application started two decades back, with concentrates on the natural marvels emerging from nourishment borne and soil borne pathogenic organisms in the sustenance and farming ventures ${ }^{2}$. Chitosan and its derivatives have shown impressive enthusiasm because of their antimicrobial and antifungal activities. The antibacterial activity of chitosan is affected by different components that depend mainly on the different types of chitosan, the dimension of chitosan polymerization and a part of its other physicochemical properties. The antibacterial activity of chitosan is affected by different components, including the kinds of microorganisms, $\mathrm{pH}$, dissolvable and sub-nuclear mass ${ }^{3,4}$. This found antimicrobial properties of chitosan were improved by stacking chitosan with different metals. The removal of the acetate ions from the chitosan microspheres was set up for controlled landing of dynamic antimicrobial administrators, for instance, amoxicillin antibiotics and metronidazole in the gastric pit ${ }^{5}$. Chitosan and its subsidiaries additionally have natural implementations in the field of antimicrobial and biomedical applications, for example, expansive range antimicrobial movement ${ }^{6-10}$. In winemaking, it tends to be utilized as a fining operator, additionally avoiding waste. In industry, it very well may be utilized in a self-recuperating polyurethane paint covering. In medication, it is valuable in swathes to lessen draining and as an antibacterial specialist; it can likewise be utilized to help convey sedates through the skin.

\section{EXPERIMENTAL STUDY MATERIALS AND MTHODS}

The crab shell via chitin was prepared from the dead and dried crabs, which were collected from the fish market in the locality. The flesh was removed and the exoskeleton was crushed and made into fine powder by commercial mixer. The powder was then transferred to a sterile beaker and left for air-drying in room temperature. Chitosan was obtained from the crab shell in the form of chitin. The run of the mill generation of chitosan from shellfish shell for the most part comprises of three fundamental processes, which include the demineralization, deproteinization and deacetylation. The shells were demineralized by disrupting reliably with $5 \% \mathrm{Hcl}$ at the extent of 1:15 ( $w / v$, shell to plan) $36 \mathrm{~h}$ at room temperature. The demineralized shells were treated with $5 \% \mathrm{NaOH}$ course of action at the extent of shell to of 1:10 $(\mathrm{w} / \mathrm{v})$ at $90-95^{\circ} \mathrm{C}$ for 5 hours. The deproteinized removed shells were isolated and washed with fixture water until $\mathrm{NaOH}$ was emptied absolutely, by then dried medium-term in an oven at $55-60^{\circ} \mathrm{C}$. The shells were filtered and washed with deionised water until wound up objective. By then de acetylating of the chitosan was then completed by hydrolyzing with $80 \% \mathrm{NaOH}$ at the extent of 1:20 (w/v, chitin to dissolvable) at $90-95^{\circ} \mathrm{C}$ for 5 hours. The obtained extract was washed with water until it ended up unprejudiced and dried medium-term at $55-60 \div \mathrm{C}$. In the arranging of chitosan $1.0 \%(\mathrm{w} / \mathrm{v})$ chitosan were dispersed in a $1.0 \%(\mathrm{v} / \mathrm{v})$ acidic destructive course of action. Chitosan activity was procured and disrupted over night with a stirrer and autoclaved at $120^{\circ} \mathrm{C}$ for 15 minutes at 15 -PSI weight ${ }^{11}$.

\section{Preparation of Chitosan nanoparticles}

Chitosan nanoparticles were produced by adding $1 \mathrm{ml}$ of tipolyphosphate (TPP) solution $(0.25 \%)$ to $4 \mathrm{ml}$ of chitosan solution under continuous mixing. The obtained nanoparticles was subjected to centrifugation at $5000 \mathrm{rpm}$ for $30 \mathrm{~min}$. The suspended supernatants were separated, and the chitosan nanoparticles was comprehensively treated with distilled water to 
remove any agents such as sodium hydroxide and then freeze- dried before further examination.

Characterization of the chitosan nanoparticles

The chitosan nanoparticles where characterized by following techniques. The ash content was measured using muffle furnace and the FTIR spectra of the chitosan nanoparticle was taken with potassium bromide pellet on JASCO FT/IR- 4000 series. The X-ray powder diffraction methods showed the patterns of chitosan nanoparticles were obtained by X-ray diffractometer. The X-ray source was CuK radiation ( $30 \mathrm{KV}, 30 \mathrm{~mA}$ ). Samples were scanned at a scanning rate of $5 \% \mathrm{~min}$. The samples for the observation of the size and morphology was prepared by drop coating the chitosan solution and was fixed with glutaraldehyde and formaldehyde solution and the surface morphology was identified by TEM (JOEL 2000 FR MARK II, Tokyo, Japan)

\section{Assay for antibacterial activity}

$1 \mathrm{ml}$ of the microorganism innoculums to be tested was added to $0.9 \mathrm{ml}$ chitosan solution ( 0.1 or $1 \% \mathrm{w} / \mathrm{v}$ ) in $0.1 \mathrm{M}$ sodium acetate buffer with a $\mathrm{PH} 6.5$ under aseptic conditions. The mixture was incubated in orbital shaker for $1 \mathrm{~h}$ at $37^{\circ} \mathrm{C}$, and the number of viable cells was calculated. To get the microorganism suspension, a loopful of inoculums from the agar plates with sterile tampon into clean saline $(0.9 \%)$ solution, at that point weakened to $10^{5}-10^{-6}$ settlement $/ \mathrm{ml}$. Test arrangements were autoclaved at $121^{\circ} \mathrm{C}$ for 15 min. One milliliter of test arrangements and 9 $\mathrm{ml}$ autoclaved supplement agar were filled in the autoclaved petri-dishes, cooled, one ring of microorganism suspension was drawn as line on cooled supplement agar, Minimum inhibitory concentration was carried out (MIC) ${ }^{12}$.

\section{RESULTS AND DISCUSSIONS}

Sulphated ash studies and morphology of the chitosan nanoparticles

The powder substance of the separated chitosan from the crab shell was evaluated by utilizing the mute heater. The powder estimation is a marker of the viability of the demineralization venture for expulsion of $\mathrm{CaCO} 3$. Disposal of the demineralization brought about items having 31$36 \%$ of the fiery remains ${ }^{13}$. The removed crab shell squander had $35.27 \%$ fiery remains content. The fiery remains substance of the chitosan acquired in this examination was run from $0.36 \%$ to $4.25 \%$ with huge contrast. The Table 1 demonstrates the substance of the dampness, sulphated cinder and the yield. The outcomes appeared in the (Fig. 1) demonstrates the remaining movement of the chitosan extricate when treated with different groupings of $\mathrm{HCl}$. The qualities demonstrated that $4 \%$ and $3 \% \mathrm{HCl}$ can adequately lessen the fiery debris substance of the got chitin up to $0.36 \%$ and $0.50 \%$, separately. A few polluting influences, for example, the left over fiery remains may influence the solvency, subsequently adding to bring down

Table 1. Sulphated ash content analysis of the extracted crab shell waste

\begin{tabular}{ll}
\hline \multicolumn{1}{c}{ Parameters } & Value \\
\hline a. Water content (\%) & 60.56 \\
b. Ash content (\%) & 65.46 \\
c. Recovery (\%) & 70.45 \\
\hline
\end{tabular}

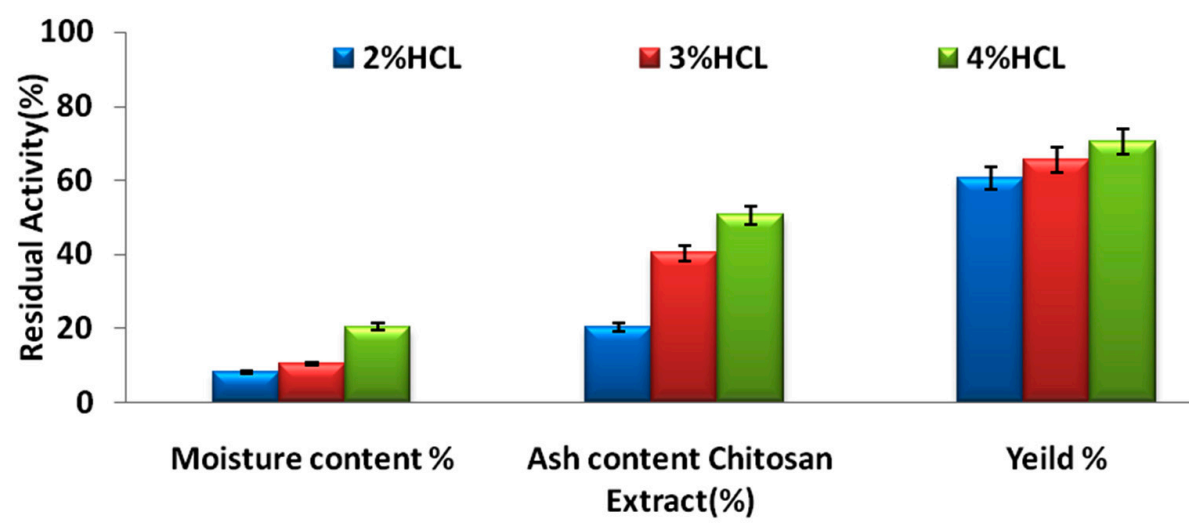

Fig.1. The residual activity of Chitosan extract with various concentration of hydrochloric acid 
thickness, or can influence other progressively last items ${ }^{14}$.

Fourier- transform infrared spectroscopy analysis of chitosan

Chitosan nanoparticles were prepared based on the ionic gelation of chitosan with tripolyphosphate anions. FTIR studies of chitosan nanoparticles were performed to characterize the chemical structure of nanoparticles. FTIR spectra of chitosan nanoparticle are shown in the (Fig. 2). A band at $3421 \mathrm{~cm}-{ }^{1}$ corresponds to the combined peaks of the $\mathrm{NH}_{2}$ and $\mathrm{OH}$ group stretching vibration in chitosan. The obtained wavelength of $1639 \mathrm{~cm}^{-1}$ was identified as $\mathrm{CONH}_{2}$ group. The formed peak shows high percentage of deacetylation of the chitosan. A marginal shift ranges from 3421- 3745 $\mathrm{cm}^{-1}$, and the crest is prominent in the chitosan nanoparticles, which indicate that the hydrogen bonding is increased. The intensities of the group at $1639 \mathrm{~cm}^{-1}$ and $\left(\mathrm{NH}_{2}\right)$ group shows the ammonium groups are cross linked with tripolyphosphate (TPP) molecules ${ }^{15}$. Thus it shows that polyphosporic group of sodium polyphosphate interacts with ammonium ion groups of chitosan, which serves to enhance the intermolecular interaction in chitosan nanoparticles ${ }^{16}$.

\section{X-Ray Diffraction (XRD) pattern of chitosan nanoparticles.}

X-Ray powder diffraction patterns of chitosan nanoparticles are shown in (Fig. 3). There is one in number crest in the diffractogram of chitosan at $2 \theta$ at $35^{\circ}$ demonstrating the elevated level of crystallinity of revealed the orientation and crystalline nature of nanoparticles that agreement with the International Center of Diffraction Data card (JCPDS-80-1916) to confirm the formation of a crystalline monoclinic structure chitosan, their precious stone cross section consistent $\alpha$ relating to 17.000 . The XRD of chitosan nanoparticles is normal for a formless polymer. Chitosan nanoparticles are included thick system structure of interpenetrating polymer fastens cross

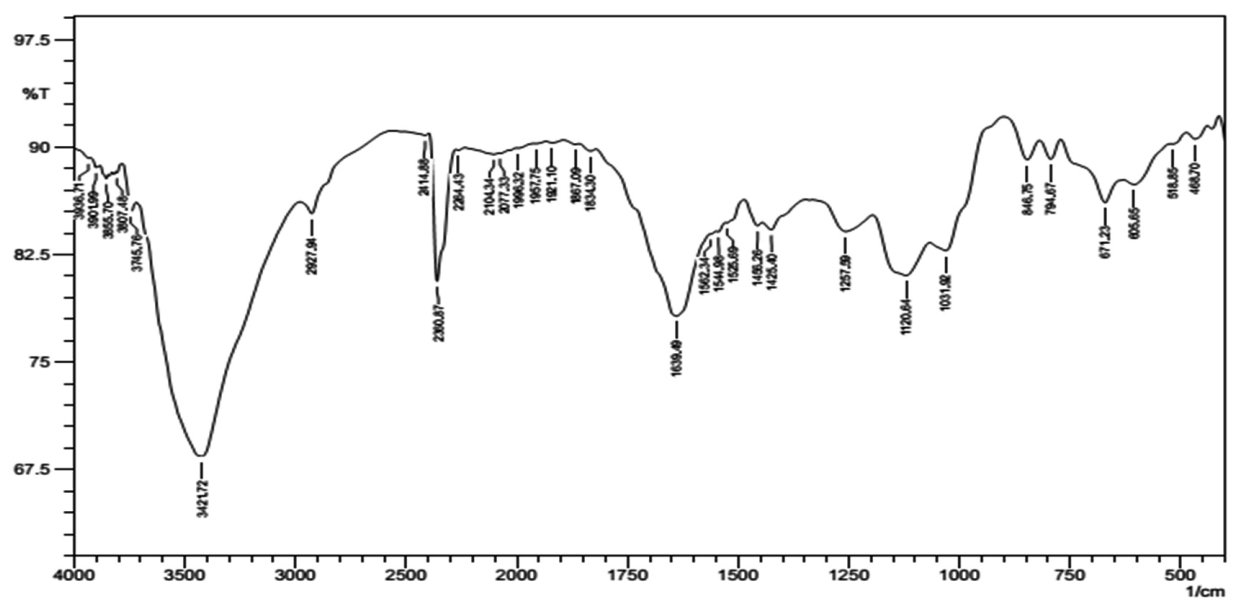

Fig. 2. FTIR spectra of the chitosan nanoparticle

Table 2. Shows the MIC of chitosan nanoparticles conjugated with antibiotics towards th microorganisms in water and acetic acid

\begin{tabular}{lcccc}
\hline Microorganisms & \multicolumn{4}{c}{ Zone of inhibition } \\
\cline { 2 - 5 } & $\begin{array}{c}\text { Chitosan } \\
\text { Np with } \\
\text { Amoxicillin }\end{array}$ & $\begin{array}{c}\text { Chitosan } \\
\text { Np with } \\
\text { Ampicillin }\end{array}$ & $\begin{array}{c}\text { Chitosan } \\
\text { Np with } \\
\text { ciprofloxacin }\end{array}$ & $\begin{array}{c}\text { Chitosan } \\
\text { Np with } \\
\text { Erythromycin }\end{array}$ \\
\hline $\begin{array}{l}\text { E. coli } \\
\text { Staplylococcus } \\
\text { aureus }\end{array}$ & $180 \mathrm{~mm}$ & $20 \mathrm{~mm}$ & $250 \mathrm{~mm}$ & $10 \mathrm{~mm}$ \\
\hline Journal of Pure and Applied Microbiology & $150 \mathrm{~mm}$ & $20 \mathrm{~mm}$ & $7 \mathrm{~mm}$ \\
\end{tabular}


connected to one another by tripolyphosphate anions. The XRD ensured the more prominent confusion in the chain arrangement in the nanoparticles after crosslinks.

\section{Antibacterial activity}

The chitosan obtained from the crab shells dissolves in the corrosive media, the settling of the chitosan arrangement becomes corrosive, and when the expansion of bacterial deferment to the chitosan nanoparticles could be easily isolated in bacterial suspension after slight stun for a decent scattering. Microbes can hold fast to the outside of the chitosan and chitosan nanoparticles essentially in brief time of 30 mins. Table 2 demonstrates the MIC of chitosan nanoparticles conjugated with antibiotic against different microorganisms in water and $0.25 \%$ acidic corrosive. The action of the chitosan nanoparticles demonstrated the antibacterial activity against the gram positive and gram-negative microscopic organisms. The (Fig. 4) shows the antibiotics conjugated with chitosan nanoparticles, against $S$. aureus and $E$. coli.

The Antimicrobial activity of the chitosanconjugated nanoparticles showed that the ciproflaxin had enhanced activity on the bacterial strains. The ciproflaxin antibiotics had shown enhanced activity towards the E.coli growth when measured in the spectrophotometer when compared to the S.aureus. The Fig. 4 and 5 Shows the optical density was measured at a wavelength of $600 \mathrm{~nm}$ where the concentration of $50 \mu \mathrm{g}$ shown a high value for the growth of the

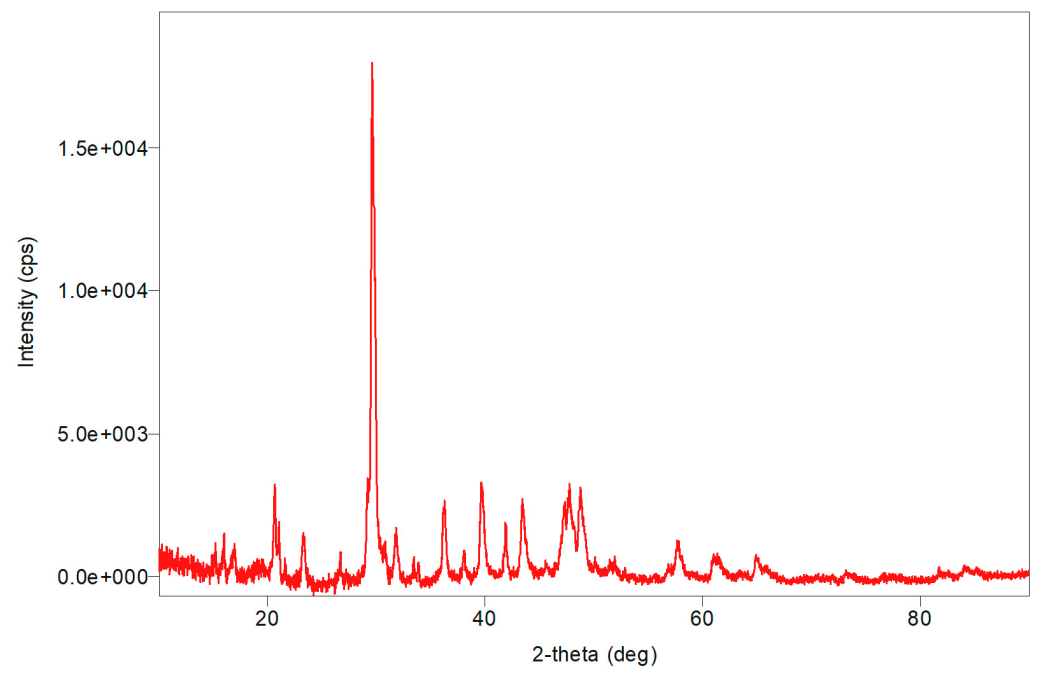

Fig. 3. X-Ray powder diffraction patterns of chitosan nanoparticle.



Fig. 4. The growth curve showing the various concentration of ciproflaxin conjugated chitosan nanoparticles and its effect on E.coli. 
E.coli rather than the S.aureus. The conjugated chitosan nanoparticles show a similar absorbance with that of the controls which is the antibiotic ciproflaxin alone. The crude extract of the chitosan nanoparticles showed that the values are similar to the commercial available antibiotics. The pure chitosan if purified is used can enhance the activity of the chitosan towards the pathogens.

Since chitosan is only soluble in acidic media, the precipitation of chitosan solution in acetic acid occurred upon addition to bacterial suspension, while chitosan nanoparticles could be well distributed in bacterial suspension after a slight shock for a nice dispersion. Bacteria can adhere to the surface of chitosan and chitosan nanoparticles significantly in short time of just 30min; thus chitosan and chitosan nanoparticles exhibit antibacterial activity ${ }^{17}$. Chitosan possess antimicrobial activity against a number of Gram-negative and Gram-positive bacteria. The antibacterial activity of chitosan nanoparticles and copper loaded nanoparticles were compared with that of chitosan in distilled water or $0.25 \%$ acetic acid, respectively ${ }^{18,19}$.

\section{Microscopic study using TEM}

Transmission electron microscopy (TEM) was used to investigate the surface morphology of chitosan nanoparticles. Micrograph of (Fig. 7) shows that chitosan nanoparticles has uniform, very well capped particle structure less than $120 \mathrm{~nm}$ in size. There is no agglomeration of nanoparticles (may be due to the presence of the chitosan as capping agent) and the surface was somewhat rough. The present study showed that the morphology as a smooth and size was $100 \mathrm{~nm}^{20}$. The TEM revealed nanoparticles with average size of $90 \mathrm{~nm}$ with narrow size distribution. In another study chitosan/DNA, nanoparticles were prepared through simple electrostatic interaction between the cationic chitosan and anionic DNA. Showed that the TEM images shows the chitosan chain dimension of around $20-40 \mathrm{~nm}$, consistent with a hydrodynamic radius of $15.8 \mathrm{~nm}$.

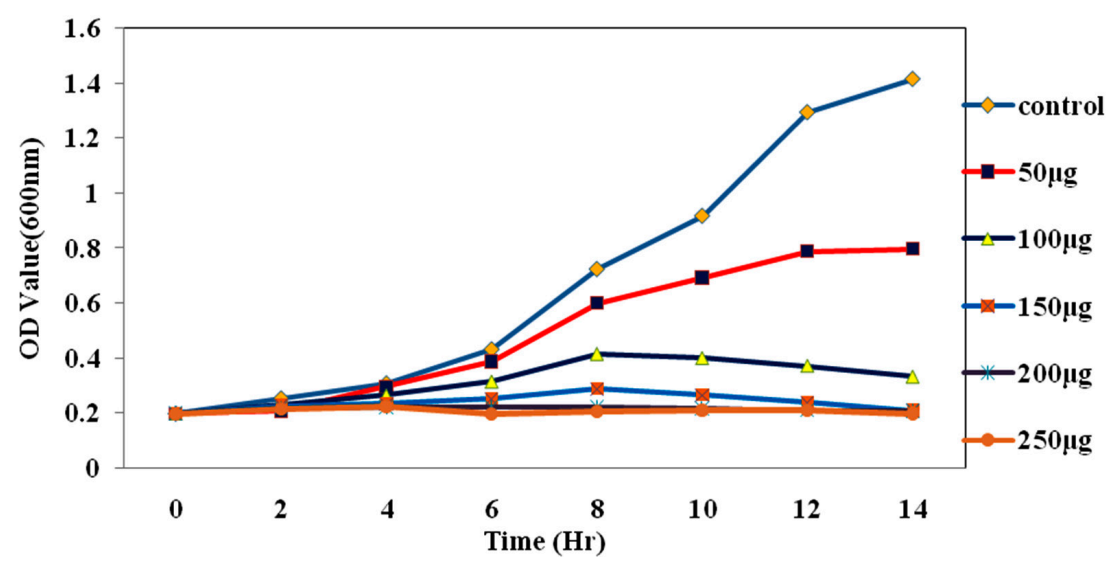

Fig. 5. The growth curve showing the various concentration of ciproflaxin conjugated chitosan nanoparticles and its effect on S.aureus.



Fig. 6. Well diffusion assay of the chitosan nanoparticles conjugated with antibiotics towards S.aureus (P1) and E.coli (P2).

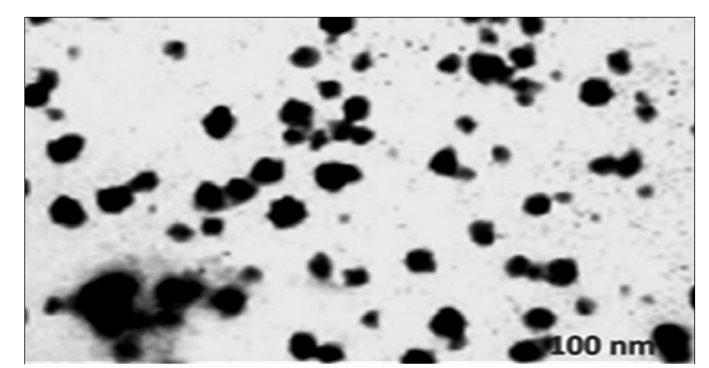

Fig. 7. TEM micrograph showing the size of the chitosan nanoparticles. 


\section{CONCLUSIONS}

The study showed that the chitosan obtained from the extracts crab shell waste showed that the positive face charges improves the strength in the presence of biological cations and improve for their antibacterial activities due to the communication with negatively charged biological membranes and site specific targeting in vivo. The chitosan-loaded nanoparticles along with the antibiotics showed the high antibacterial potential against the microorganisms. The study reveals to facilitate chitosan nanoparticles to be generally used as the antimicrobial agent in medicine for their high antibacterial activity and acceptable biocompatibilities and mainly used as biopolymers and it can be used for the targeted drug delivery mechanism. The morphology of the chitosan nanoparticle showed a size of $100 \mathrm{~nm}$ that was comparatively higher when compared to previous obtained nanoparticles derived from the chitosan from the crab shells.

\section{ACKNOWLEDGEMENTS}

The authors are much thankful to the Department of Industrial Biotechnology- BIHER for providing the instrumentation facility for the work. The authors are also indebted to thank the Department Head Dr. L. Jeyanthi Rebecca for her kind help provided during the study.

\section{CONFLICTS OF INTEREST}

The authors declare that there is no conflict of interest.

\section{FUNDING}

None.

\section{AUTHOR'S CONTRIBUTION}

All authors listed have made a substantial, direct and intellectual contribution to the work, and approved it for publication.

\section{DATA AVAILABILITY}

All datasets generated or analyzed during this study are included in the manuscript.

\section{ETHICS STATEMENT}

This article does not contain any studies with human participants or animals performed by any of the authors.

\section{REFERENCES}

1. Kendra, D.F.; Hadwiger, L.A. Characterization of the smallest chitosan oligomer that is maximally antifungal to Fusarium solani and elicits pisatin formation in Pisum Sativum, Exp. Mycol. 1984; 8: 276-281. https:// doi.org/10.1016/0147-5975(84)90013-6

2. Rabea, E.I., Badawy, M.E.T., Stevens, C.V., Smagghe, G., Steurbaut, W., Chitosan antimicrobial agent: applications and mode of action. Biomacromolecules, 2003; 4: 1457-1465. https://doi.org/10.1021/ bm034130m

3. Du, W.X., Olsen, C.W., Avena-bustillos, R.J., Mchugh, T.H., Levin, C.E., Friedman, M., Storage stability and antibacterial activity against Escherichia coli 0157: $\mathrm{H} 7$ of carvacrol in edible apple films made by two different casting methods. Journal of Agricultural and Food Chemistry, 2008; 56: 3082-308. https://doi. org/10.1021/jf703629s

4. Portero, A., Remunan-Lopez, C., Criado, M.T., Alonso, M.J.J. Microencapsulation, 2002; 19: 797-809. https:// doi.org/10.1080/0265204021000022761

5. Md. Monarul Islam Md., Shah Md. Masum, Khandaker Rayhan Mahbub and Md. Zahurul Haque. Antibacterial Activity of Crab-Chitosan against Staphylococcus aureus and Escherichia coli. Journal of Advanced Scientific Research, 2011 2(4): 63-66.

6. Hui Liu, Yumin Du, Jianhong Yang, Huayue Zhu. Structural characterization and antimicrobial activity of chitosan / betaine derivative complex Carbohydrate Polymers, 2004; 55: 291-297. https:// doi.org/10.1016/j.carbpol.2003.10.001

7. Bough, W.A., Salter, W.L., Wu, A.C.M., and Perkins, B.E. Influence of manufacturing variables on the characteristics and effectiveness of chitosan products. Chemical composition, viscosity, and molecular weight distribution of chitosan products. Biotechnol. Bioeng., 1978; 20:1931. https://doi.org/10.1002/ bit. 260201208

8. No, H.K., Lee, M.Y. Isolation of Chitin from Crab Shell Waste. Journal Korean Soc. Food Nutrition, 1995; 24(1): 105-113.

9. Xu, Y.M., Du, Y.M., Effect of molecular structure of chitosan on protein delivery properties of chitosan nanoparticles. Int. J. Pharm., 2003; 250: 215-226. https://doi.org/10.1016/S0378-5173(02)00548-3

10. Knaul, J.Z., Hudson, S.M., Creber, K.A.M., Improved mechanical properties of Chitosan fibers. Journal of Appl. Polymer. Sci., 1999; 72: 1721-1731. https://doi.org/10.1002/ (SICI)1097-4628(19990624)72:13\%3C1721::AIDAPP8\%3E3.0.CO;2-V

11. Rampino A, Borgogna M, Blasi P, Bellich B, Cesaro A. Chitosan nanoparticles: preparation, size evolution and stability. Int. J. Pharm., 2013; 455(1-2): 219-28. https://doi.org/10.1016/j.ijpharm.2013.07.034

12. Ilyina A.V., V.E. Tikhonov, A.I. Albulov, V.P. Varlamov. Enzymatic preparation of acid- freewater soluble chitosan, Process. Biochem., 2000; 35(2000): 563-568. https://doi.org/10.1016/S00329592(99)00104-1

13. Jia Z., D. Shen, W. Xu. Synthesis and antibacterial activities of quaternary ammonium salt of chitosan, 
Carbohydr. Res., 2001; 333(1): 1-6. https://doi. org/10.1016/S0008-6215(01)00112-4

14. Jo C., J.W. Lee, K.H. Lee, M.W. Byun. Quality properties of pork sausage prepared with water-soluble chitosan oligomer, Meat. Sci., 2001; 59(4): 369-375. https://doi. org/10.1016/S0309-1740(01)00089-4

15 Allan, C.R., L.A. Hardwiger. The fungicidal effect of chitosan on fungi of varying cell wall composition, Exp. Mycol., 1979; 3(3): 285-287. https://doi.org/10.1016/ S0147-5975(79)80054-7

16 Qi Li, Xu Z, Jiang X, Hu C, Zou X. Preparation and antibacterial activity of chitosan Nanoparticle Carbohydr Res., 2004; 339(16): 2693-2700. https:// doi.org/10.1016/j.carres.2004.09.007
17

Ueno, K.; Yamaguchi, T.; Sakairi, N.; Nishi, N.; Tokura, S. Adv. Chitin Sci., 1997; 2: $156-161$.

18 Jeon, Y.J.; Park, P.J.; Kim, S.K. Carbohydr. Polym., 2001; 44: 71-76. https://doi.org/10.1016/S0144$8617(00) 00200-9$

19 Qi Li, Xu Z, Jiang X, Hu C, Zou X. Preparation and antibacterial activity of chitosan Nanoparticle Carbohydr Res., 2004; 339(16): 2693-2700. https:// doi.org/10.1016/j.carres.2004.09.007

20. Nimesh S, Saxena A, Kumar A, Chandra R. Improved transfection efficiency of Chitosan-DNA complexes employing reverse transfection. J Appl Polym Sci., 2012; 124: 1771-1777. https://doi.org/10.1002/ app.35179 